\title{
Proximity-induced pseudogap in mesoscopic superconductor/normal-metal bilayers
}

\author{
Guo-Qiao Zha, ${ }^{1,2}$ Lucian Covaci, ${ }^{1}$ Shi-Ping Zhou, ${ }^{1,2}$ and F. M. Peeters ${ }^{1}$ \\ ${ }^{1}$ Departement Fysica, Universiteit Antwerpen, Groenenborgerlaan 171, B-2020 Antwerpen, Belgium \\ ${ }^{2}$ Department of Physics, Shanghai University, Shanghai 200444, China \\ (Received 14 July 2010; revised manuscript received 1 September 2010; published 5 October 2010)
}

\begin{abstract}
Recent scanning tunneling microscopy (STM) measurements of the proximity effect in $\mathrm{Au} / \mathrm{La}_{2-x} \mathrm{Sr}_{x} \mathrm{CuO}_{4}$ and $\mathrm{La}_{1.55} \mathrm{Sr}_{0.45} \mathrm{CuO}_{4} / \mathrm{La}_{2-x} \mathrm{Sr}_{x} \mathrm{CuO}_{4}$ bilayers showed a proximity-induced pseudogap [O. Yuli, I. Asulin, Y. Kalcheim, G. Koren, and O. Millo, Phys. Rev. Lett. 103, 197003 (2009)]. We describe the proximity effect in mesoscopic superconductor/normal-metal bilayers by using the Bogoliubov-de Gennes equations for a tightbinding Hamiltonian with competing antiferromagnetic and $d$-wave superconductivity orders. The temperaturedependent local density of states is calculated as a function of the distance from the interface. Bound state due to both $d$-wave and spin-density wave gaps are formed in the normal metal for energies less than the respective gaps. If there is a mismatch between the Fermi velocities in the two layers we observe that these states will shift in energy when spin-density wave order is present, thus inducing a minigap at finite energy. We conclude that the STM measurement in the proximity structures is able to distinguish between the two scenarios proposed for the pseudogap (competing or precursor to superconductivity).
\end{abstract}

DOI: $10.1103 /$ PhysRevB.82.140502

PACS number(s): 74.45.+c, 74.72. $-\mathrm{h}$

One of the most important issues in cuprate hightemperature superconductors is the existence of a pseudogap (PG) phase in the underdoped region. ${ }^{1,2}$ It has received heavy debate about its nature as its origin may hold key information regarding the pairing mechanism in these materials. One paradigm assumes that the PG is only a manifestation of a competing or coexisting order of superconductivity and it has no direct relation to the pairing. ${ }^{3-5}$ However, other scenarios regard the $\mathrm{PG}$ as a superconducting precursor state ${ }^{6-11}$ with close relation to Cooper pairing. Despite many studies on the subject, it remains unclear whether the PG phenomenon is related to superconductivity or not.

Very recently, the temperature evolution of the proximity-induced gap in $\mathrm{Au} / \mathrm{La}_{2-x} \mathrm{Sr}_{x} \mathrm{CuO}_{4}$ and $\mathrm{La}_{1.55} \mathrm{Sr}_{0.45} \mathrm{CuO}_{4} / \mathrm{La}_{2-x} \mathrm{Sr}_{x} \mathrm{CuO}_{4}$ bilayers was reported by Yuli et al. ${ }^{12}$ using scanning tunneling microscopy (STM) and scanning tunneling spectroscopy (STS) measurement to examine whether unique spectral properties associated with pairing are present above the superconducting transition temperature $T_{c}$ in the PG temperature regime. For bilayers comprising an underdoped $\mathrm{La}_{2-x} \mathrm{Sr}_{x} \mathrm{CuO}_{4}$ (LSCO) film, there exists a smooth evolution of the superconductor proximity gap into a proximity-induced $\mathrm{PG}$, as the temperature was raised above $T_{c}$. In contrast, the proximity gap disappeared close to $T_{c}$ in bilayers comprising an overdoped LSCO layer. They claimed that the similar spatial dependence of the proximityinduced PG and the superconductor proximity gap indicates that the origin of the PG is related to superconductivity. How to understand these behaviors theoretically is still a challenging question. The proximity effect between a superconductor and a normal $(\mathrm{N})$ metal has been thoroughly studied using various techniques. ${ }^{13-17}$ However, an investigation of the proximity effect in normal-metal/superconductor bilayers above $T_{c}$ is still lacking.

In the present Rapid Communication, we use numerical solutions of the Bogoliubov-de Gennes (BdG) equations based on a model Hamiltonian with competing $d$-wave superconducting (DSC) and antiferromagnetic (AFM) orders at finite temperature and examine the proximity-induced gap in mesoscopic normal-metal/superconductor bilayers. In cuprate superconductors, the spin-density wave (SDW) order with stripe modulation and the accompanying charge order may emerge at high-temperature $T$ and could exist above the DSC transition temperature $T_{c}{ }^{18}$ Our numerical analysis focuses on the interplay between DSC and SDW orders when in proximity with the metal as well as the local density of states (LDOS) as a function of temperature and distance from the interface. States induced in the normal-metal region depend on both DSC and SDW orders present in the superconducting side and on the Fermi velocity mismatch at the interface. Our results provide important insight into the interpretation of recent STM experiments. ${ }^{12}$

In order to describe the superconductor/normal-metal bilayers we use the tight-binding extended Hubbard Hamiltonian by assuming that the on-site repulsion $U_{\mathbf{i}}$ is responsible for the competing AFM order and the nearest-neighbor attraction $V_{\mathrm{ij}}$ for the DSC pairing in the superconducting region,

$$
\begin{aligned}
H= & -\sum_{\langle\mathbf{i j}\rangle, \sigma} t_{\mathbf{i j}} c_{\mathbf{i} \sigma}^{\dagger} c_{\mathbf{j} \sigma}+\sum_{\mathbf{i}, \sigma}\left(U_{\mathbf{i}}\left\langle n_{\mathbf{i} \sigma}\right\rangle-\mu\right) c_{\mathbf{i} \sigma}^{\dagger} c_{\mathbf{i} \sigma} \\
& +\sum_{\langle\mathbf{i} \mathbf{j}\rangle}\left(\Delta_{\mathbf{i j}} c_{\mathbf{i} \uparrow}^{\dagger} c_{\mathbf{j} \downarrow}^{\dagger}+\Delta_{\mathbf{i j}}^{*} c_{\mathbf{j} \downarrow} c_{\mathbf{i} \uparrow}\right),
\end{aligned}
$$

where $t_{\mathrm{ij}}=t$ are the nearest-neighbor hopping integral. $c_{\mathbf{i} \sigma}$ $\left(c_{\mathbf{i} \sigma}^{\dagger}\right)$ are destruction (creation) operators for electron of spin $\sigma, n_{\mathbf{i} \sigma}=c_{\mathbf{i} \sigma}^{\dagger} c_{\mathbf{i} \sigma}$ is the number operator, and $\mu$ is the chemical potential determining the averaged electron density $\bar{n}$. The SDW and DSC orders have the following definitions, respectively: $\Delta_{\mathbf{i}}^{\mathrm{SDW}}=U_{\mathbf{i}}\left\langle c_{\mathbf{i} \uparrow}^{\dagger} c_{\mathbf{i} \uparrow}-c_{\mathbf{i} \downarrow}^{\dagger} c_{\mathbf{i} \downarrow}\right\rangle$ and $\Delta_{\mathbf{i j}}=V_{\mathbf{i j}} \chi_{i j}$, where $\chi_{i j}=\left\langle c_{\mathbf{i} \uparrow} c_{\mathbf{j} \downarrow}-c_{\mathbf{i} \downarrow} c_{\mathbf{j} \uparrow}\right\rangle / 2$ is the pair-correlation function. Using the Bogoliubov transformation, $c_{\mathbf{i} \sigma}=\sum_{n}\left[u_{\mathbf{i} \sigma}^{n} \gamma_{n \sigma}-\sigma v_{\mathbf{i} \sigma}^{n *} \gamma_{n \bar{\sigma}}^{\dagger}\right]$, the Hamiltonian in Eq. (1) can be diagonalized by solving the resulting $\mathrm{BdG}$ equations self-consistently, 


$$
\sum_{\mathbf{j}}^{N}\left(\begin{array}{cc}
\mathcal{H}_{\mathbf{i} \mathbf{j} \sigma} & \Delta_{\mathbf{i j}} \\
\Delta_{\mathbf{i} \mathbf{j}}^{*} & -\mathcal{H}_{\mathbf{i} \mathbf{j} \bar{\sigma}}^{*}
\end{array}\right)\left(\begin{array}{c}
u_{\mathbf{j} \sigma}^{n} \\
v_{\mathbf{j} \bar{\sigma}}^{n}
\end{array}\right)=E_{n}\left(\begin{array}{c}
u_{\mathbf{i} \sigma}^{n} \\
v_{\mathbf{i} \bar{\sigma}}^{n}
\end{array}\right),
$$

where $\mathcal{H}_{\mathbf{i j} \sigma}=-t_{\mathbf{i j}}+\left[U_{\mathbf{i}}\left\langle n_{\mathbf{i} \bar{\sigma}}\right\rangle-\mu\right] \delta_{\mathrm{ij}}$. Using open boundary conditions we obtain the eigenvalues $\left\{E_{n}\right\}$ with eigenvectors $\left\{u^{n}, v^{n}\right\}$.

The self-consistent conditions are

$$
\begin{gathered}
\left\langle n_{\mathbf{i} \uparrow}\right\rangle=\sum_{n=1}^{2 N}\left|\mathbf{u}_{\mathbf{i}}^{n}\right|^{2} f\left(E_{n}\right), \\
\left\langle n_{\mathbf{i} \downarrow}\right\rangle=\sum_{n=1}^{2 N}\left|\mathbf{v}_{\mathbf{i}}^{n}\right|^{2}\left[1-f\left(E_{n}\right)\right], \\
\Delta_{\mathbf{i j}}=\sum_{n=1}^{2 N} \frac{V_{\mathbf{i j}}}{4}\left(\mathbf{u}_{\mathbf{i}}^{n} \mathbf{v}_{\mathbf{j}}^{n *}+\mathbf{v}_{\mathbf{i}}^{n *} \mathbf{u}_{\mathbf{j}}^{n}\right) \tanh \left(\frac{E_{n}}{2 k_{B} T}\right),
\end{gathered}
$$

where $f(E)=\left(e^{E / k_{B} T}+1\right)^{-1}$ is the Fermi-Dirac distribution function. The DSC pair-correlation function is defined at site $i$ as $\chi_{\mathbf{i}}^{D}=\left(\chi_{\mathbf{i}+\mathbf{e}_{\mathbf{x}}, \mathbf{i}}+\chi_{\mathbf{i}-\mathbf{e}_{\mathbf{x}}, \mathbf{i}}-\chi_{\mathbf{i}, \mathbf{i}+\mathbf{e}_{\mathbf{y}}}-\chi_{\mathbf{i}, \mathbf{i}-\mathbf{e}_{\mathbf{y}}}\right) / 4$, where $\mathbf{e}_{\mathbf{x},(\mathbf{y})}$ denotes the unit vector along the $x(y)$ direction. Throughout this work, the distance is measured in units of the lattice constant $a$ and the energy is scaled to $t$. In the numerical calculations, we take $k_{B}=a=t=1$ for simplicity. The averaged electron density is chosen as $\bar{n}=0.9$, corresponding to the underdoped level $\delta=0.1$. For an appropriate initial orderparameter profile, the Hamiltonian is numerically diagonalized and the obtained electron wave functions are used to calculate the new parameters for the next iteration step. The solution is found when the relative error in the gap function between successive iterations is less than the desired accuracy.

The $d I / d V$ at energy $E$ and position $\mathbf{i}$ can be written as

$$
\rho_{\mathbf{i}}(E)=\sum_{n=1}^{2 N}\left[\left|\mathbf{u}_{\mathbf{i}}^{n}\right|^{2} f^{\prime}\left(E-E_{n}\right)+\left|\mathbf{v}_{\mathbf{i}}^{n}\right|^{2} f^{\prime}\left(E+E_{n}\right)\right],
$$

where $f^{\prime}(E)$ is the derivative of the Fermi distribution function. $\rho_{\mathrm{i}}(E)$ is proportional to the local differential tunneling conductance which could be measured by STM experiments.

First, we consider mesoscopic superconductor/normalmetal bilayers with sizes of $N_{x} \times N_{y}=32 \times 32(12 \times 32)$ for the superconducting (normal) region. The spatial variation in (a) the DSC pair-correlation function $\chi_{i}^{D}$ and (b) the staggered magnetization $M_{i}^{s}$ along the $x$ axis for various temperatures $T$ are plotted in Fig. 1 for chosen parameter values: $U=2.5 t, V=1.2 t$, and $t^{\prime}=0$. Here, the staggered magnetization of the induced AFM or SDW order is defined as $M_{i}^{s}=(-1)^{i}\left(n_{i \uparrow}-n_{i \downarrow}\right)$. Figure 2 shows the DSC and SDW correlation functions for the same pairing potentials but for a different next-nearest-neighbor hopping $t^{\prime}=-0.2 t$ in order to properly account for the band structure of high- $T_{c}$ cuprates. One can easily see that, for both cases, the $y$-oriented stripe modulations of DSC and coexisting SDW orders are present at lower temperatures. Note that, the oscillation periods decrease with increasing the next-nearest-neighbor hopping strength while the period of the SDW is almost half of that of

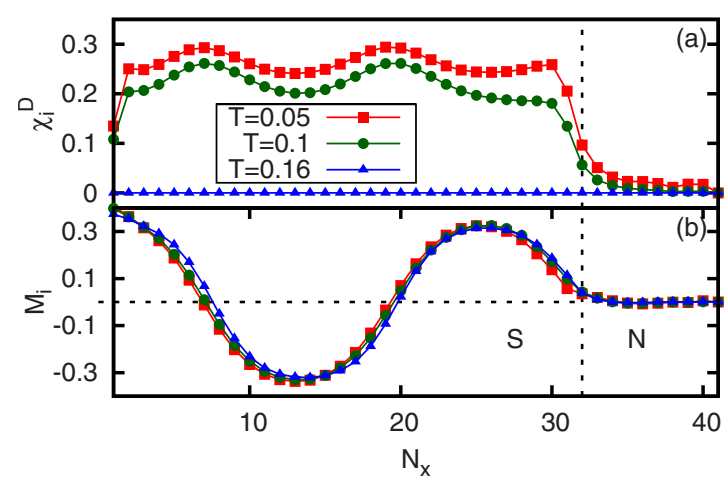

FIG. 1. (Color online) The spatial variation in (a) DSC paircorrelation function $\chi_{i}^{D}$ and (b) staggered magnetization $M_{i}^{s}$ as a function of $N_{x}$ for different temperatures $T$ in a mesoscopic normalmetal/superconductor bilayer. "S" and "N" denote, respectively, the superconducting region and normal metal. The parameter values are: $U=2.5, \bar{n}=0.9$, and $t^{\prime}=0$.

the DSC. Moreover, the periodicity of the strip-modulated DSC and SDW orders for $t^{\prime}=0$ seem not to be sensitive to $T$. When the temperature increases, the DSC order is suppressed and finally disappears at $T_{c}$. Further increasing $T$, the staggered magnetization decreases to zero at the Neél temperature $T_{N}=0.3$. In this case the nature of the pseudogap is the competing SDW order present only at low doping and higher temperature. This coexistence could be the reason for the modulation of the superconducting gap observed in STM experiments on underdoped cuprates. ${ }^{19}$

Shown in Figs. 1 and 2, both DSC [panel (a)] and SDW [panel (b)] leak into the normal region due to the proximity effect. The usual exponential decay is observed with a leaking distance which is of the order of the lattice constant. Similar to bilayers made of conventional SC and normal metals, bound states will be induced in the normal region for energies less than the superconducting gap. In order to see this we plot in Fig. $3 \rho_{i}$ around the SC/normal-metal interface. Similar to Ref. 18 we find that in the superconducting region as the temperature is increased above $T_{c}$ a gap still exists due to the coexisting SDW order. Unfortunately, mainly due to the discreteness of the energy spectrum and

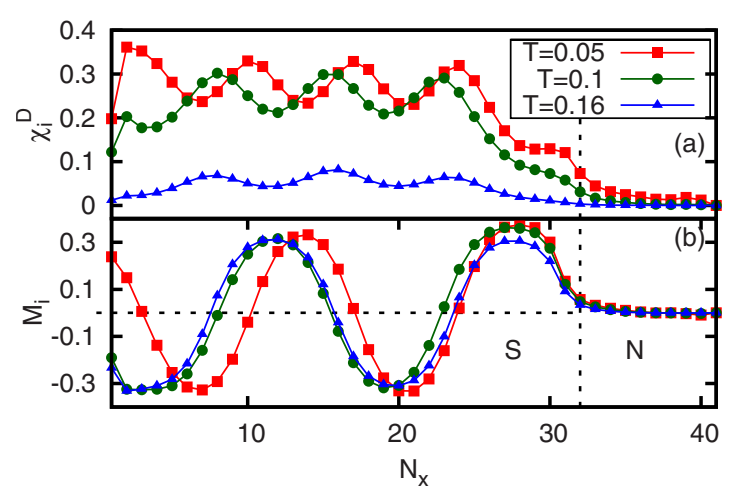

FIG. 2. (Color online) The spatial variation in (a) $\chi_{i}^{D}$ and (b) $M_{i}^{s}$ as a function of $N_{x}$ for different temperatures $T$ in a mesoscopic normal-metal/superconductor bilayers. The parameter values are: $U=2.5, \bar{n}=0.9$, and $t^{\prime}=-0.2$. 


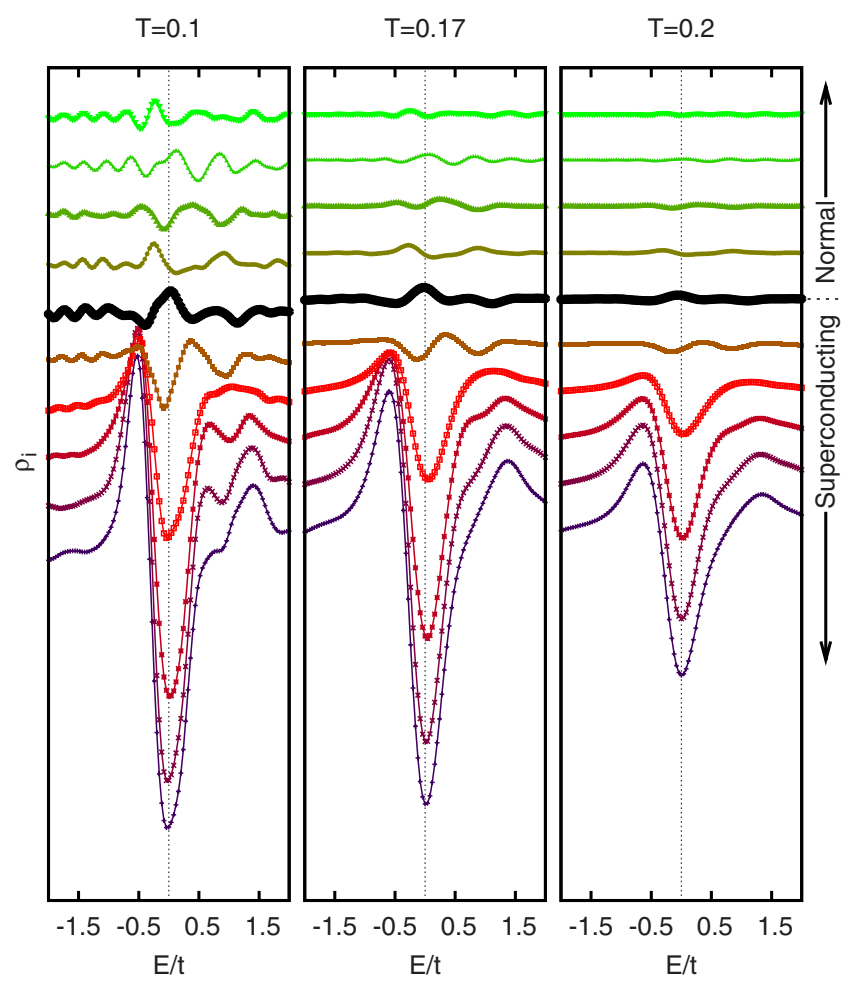

FIG. 3. (Color online) The $d I / d V$ for positions around the interface for $t^{\prime}=-0.2 t$ with $T=0.1, T_{c}=0.17$, and $T=0.2$. The other parameter values are: $U=2.5$ and $\bar{n}=0.9$. The thick black line represents the $\rho_{i}$ at the interface while lower (upper) lines are in the SC (normal) region. $\rho_{i}$ calculated for the normal state is subtracted for all lines in order to suppress oscillations due to the interface with vacuum.

the finite-temperature smearing, we cannot draw meaningful conclusions from $\rho_{i}$ calculated in the normal-metal region. The main observation from Fig. 3 is the modification of the $d I / d V$ curve in the normal-metal region for energies below the DSC or SDW gaps due to subgap bound states. Because the width of the normal region is larger than the DSC/SDW coherence length, the induced minigap is below the resolution of the calculation and thus unobservable.

In order to better understand the formation of bounds states in the normal region we performed a slightly different calculation. With the insight given by the self-consistent calculation done on a smaller sized system, we calculated the LDOS for a bilayer of size $N_{x} \times N_{y}=300 \times 500$ with a normal-metal region of size $L=4 a$. While still using the same model Hamiltonian we do not recalculate the order parameters self-consistently but consider them as step functions. If we are interested only in the LDOS at the surface of the normal-metal this approximation is reasonable since all the order parameters are set to zero in the normal region while if the coherence length is small it results in a sharp drop of the order parameters at the $\mathrm{SC} / \mathrm{N}$ interface. Now instead of diagonalizing the full Hamiltonian we approximate the Green's function and hence the LDOS in terms of Chebyshev polynomials. The procedure is presented elsewhere. ${ }^{20}$

Similar to an Andreev reflection at the N/SC interface for quasiparticles with energies lower than the superconducting gap, at the N/AFM interface a peculiar reflection ( $Q$

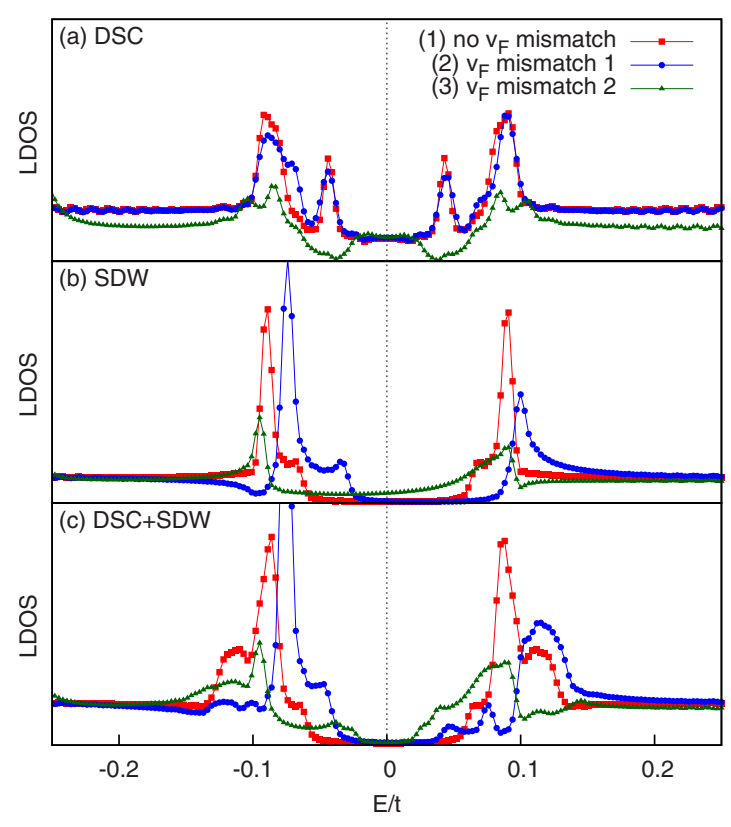

FIG. 4. (Color online) The LDOS at the surface of the normalmetal region for three choices of order parameters: (a) $\Delta^{D}=0.1 t$, $\Delta^{\mathrm{SDW}}=0.0$, (b) $\Delta^{D}=0.0, \Delta^{\mathrm{SDW}}=0.1 t$, and (c) $\Delta^{D}=0.1 t, \Delta^{\mathrm{SDW}}=0.1 t$. The lines represent three Fermi velocity mismatches at the N/SC interface: (1) no mismatch, (2) mismatch due to change in chemical potential, and (3) mismatch due to change in next-nearest-neighbor hopping. The thickness of the normal-metal region is smaller than the coherence lengths of the two order parameters. The broadening used in calculating the LDOS is $\eta=0.0005 t$.

reflection $^{21,22}$ ) occurs if the energy is less than the SDW gap. Quasiparticles with momentum $\mathbf{k}_{F}$ are scattered to states with momentum $\mathbf{k}_{F}+\mathbf{Q}$, where $\mathbf{Q}$ is defined by the AFM order. If there is a potential barrier at the interface or a mismatch in the Fermi velocity, in addition to the $\mathrm{Q}$ reflection, specular reflection also occurs thus influencing the formation of the bound states.

The main point in the STM measurement ${ }^{12}$ was the fact that the observed gap is always at zero energy whether the normal-metal layer is overdoped LSCO or Au. To simulate these conditions we consider three situations: no Fermi velocity mismatch, Fermi velocity mismatch from a shift in the chemical potential in the normal region $(\delta \mu=0.1 t)$, or Fermi velocity mismatch from a different band structure $\left(t^{\prime}=0\right.$ in the $\mathrm{SC}$ region while $t^{\prime}=-0.2 t$ in the normal region). In Fig. 4 we plot the calculated LDOS for three choices of the order parameters. First, in Fig. 4(a) only DSC $\left(\Delta_{d}=0.1 t\right)$ is considered. Subgap structures appear for all three choices of interfaces but the minigap is independent of this choice, the energies of subgap states do not shift. Second, in Fig. 4(b) only SDW $\left(\Delta_{\text {SDW }}=0.1 t\right)$ is considered. Subgap features are also observed but depending whether there is Fermi velocity mismatch or not, the bound states shift in energy. The minigap now shifts to higher or lower energies. Third, in Fig. 4(c) we consider a coexistence of DSC and SDW $\left(\Delta_{d}=0.1 t\right.$ and $\Delta_{\text {SDW }}=0.1 t$ ). In this case a minigap is still observed when the next-nearest-neighbor hopping is changed in the normal layer although the particle-hole symmetry is broken. When the chemical potential is shifted in the normal metal, the 
location of the subgap features is again shifted. This behavior is not consistent to the experimental STM findings, ${ }^{12}$ which showed that the minimum of the proximity-induced pseudogap is always at the Fermi level irrespective of the normal-metal type (overdoped LSCO or Au). In order to explain the observed proximity-induced pseudogap only scenarios which involve reflections with $\mathbf{Q}=\mathbf{0}$, e.g., fluctuating $\mathrm{SC}$ order parameters or loop current orders, ${ }^{4}$ should be relevant.

In conclusion, we have investigated the proximity effect in mesoscopic normal-metal/superconductor bilayers by numerically solving the $\mathrm{BdG}$ equations based on an effective model Hamiltonian with competing AFM and $d$-wave superconductivity interactions. The self-consistent solution gives oscillating DSC and SDW order parameters with the period of oscillations determined by the value of the next-nearestneighbor hopping amplitude. For low doping there exists a temperature range above $T_{c}$ in which only the SDW order is stable. As expected we observe in the metallic region the proximity-induced pair correlations and the localized bound states for energies less that the DSC (or SDW) gap. More importantly by using a larger system we were able to confirm the conclusion presented in Ref. 12 which argues that an SDW order above $T_{c}$ would shift the minimum gap energy if the normal metal is changed from overdoped LSCO to Au. By using a model microscopic Hamiltonian we showed that a mismatch in the Fermi velocity at the N/SC interface would shift the induced minigap. Depending on the nature of this mismatch (potential barrier or different band structure) the shift should also be observed below $T_{c}$ in the temperature range where DSC and SDW order coexist. The same conclusions should hold for $c$ axis or nodal surfaces when looking at the proximity effect. Modifications will appear in the LDOS only as broadening of the Andreev bound states (for $c$-axis orientation due to the modulation of the SDW and DSC order parameters) or presence of zero energy states [for (110) surfaces]. The shift due to Fermi velocity mismatch for the SDW scenario will not be influenced by the crystallographic orientation.

This work was supported by the Flemish Science Foundation (FWO-Vl), by Belgian Science Policy (IAP), by National Natural Science Foundation of China under Grants No. 10904089 and No. 60971053, by the Research Fund of Higher Education of China under Grant No. 20093108120005, by Shanghai Leading Academic Discipline project under Grant No. S30105, by Science and Technology Committee of Shanghai Municipal under Grant No. 09JC1406000, by Shanghai Municipal Education Committee under Grants No. shu-08053 and No. 10zz63, and by Innovation Funds of Shanghai University.
${ }^{1}$ T. Timusk and B. W. Statt, Rep. Prog. Phys. 62, 61 (1999).

${ }^{2}$ S. Hüfner, M. A. Hossain, A. Damascelli, and G. A. Sawatzky, Rep. Prog. Phys. 71, 062501 (2008).

${ }^{3}$ S. Chakravarty, R. B. Laughlin, D. K. Morr, and C. Nayak, Phys. Rev. B 63, 094503 (2001).

${ }^{4}$ C. M. Varma, Phys. Rev. B 73, 155113 (2006).

${ }^{5}$ J.-X. Li, C. Q. Wu, and D. H. Lee, Phys. Rev. B 74, 184515 (2006).

${ }^{6}$ Y. J. Uemura et al., Phys. Rev. Lett. 62, 2317 (1989).

${ }^{7}$ V. J. Emery and S. A. Kivelson, Nature (London) 374, 434 (1995).

${ }^{8}$ Z. Y. Weng, D. N. Sheng, and C. S. Ting, Phys. Rev. Lett. 80, 5401 (1998)

${ }^{9}$ J. Corson et al., Nature (London) 398, 221 (1999).

${ }^{10}$ Y. Wang, L. Li, and N. P. Ong, Phys. Rev. B 73, 024510 (2006).

${ }^{11}$ H.-H. Wen, G. Mu, H. Luo, H. Yang, L. Shan, C. Ren, P. Cheng, J. Yan, and L. Fang, Phys. Rev. Lett. 103, 067002 (2009).
${ }^{12}$ O. Yuli, I. Asulin, Y. Kalcheim, G. Koren, and O. Millo, Phys. Rev. Lett. 103, 197003 (2009).

${ }^{13}$ J. H. Chen, Phys. Rev. B 42, 3952 (1990); 42, 3957 (1990).

${ }^{14}$ J. Z. Wu, X. X. Yao, C. S. Ting, and W. K. Chu, Phys. Rev. B 46, 14059 (1992).

${ }^{15}$ J. X. Zhu and C. S. Ting, Phys. Rev. B 61, 1456 (2000).

${ }^{16}$ K. Halterman and O. T. Valls, Phys. Rev. B 65, 014509 (2001).

${ }^{17}$ L. Covaci and F. Marsiglio, Phys. Rev. B 73, 014503 (2006).

${ }^{18}$ H.-Y. Chen and C. S. Ting, Phys. Rev. B 71, 132505 (2005).

${ }^{19}$ M. Vershinin, Science 303, 1995 (2004).

${ }^{20}$ L. Covaci, F. M. Peeters, and M. Berciu, arXiv:1007.1609, Phys. Rev. Lett. (to be published).

${ }^{21}$ B. M. Andersen, I. V. Bobkova, P. J. Hirschfeld, and Y. S. Barash, Phys. Rev. B 72, 184510 (2005).

${ }^{22}$ I. V. Bobkova, P. J. Hirschfeld, and Y. S. Barash, Phys. Rev. Lett. 94, 037005 (2005). 